\title{
Growth of hepatocellular adenoma during pregnancy: A prospective study
}

\section{Graphical abstract}

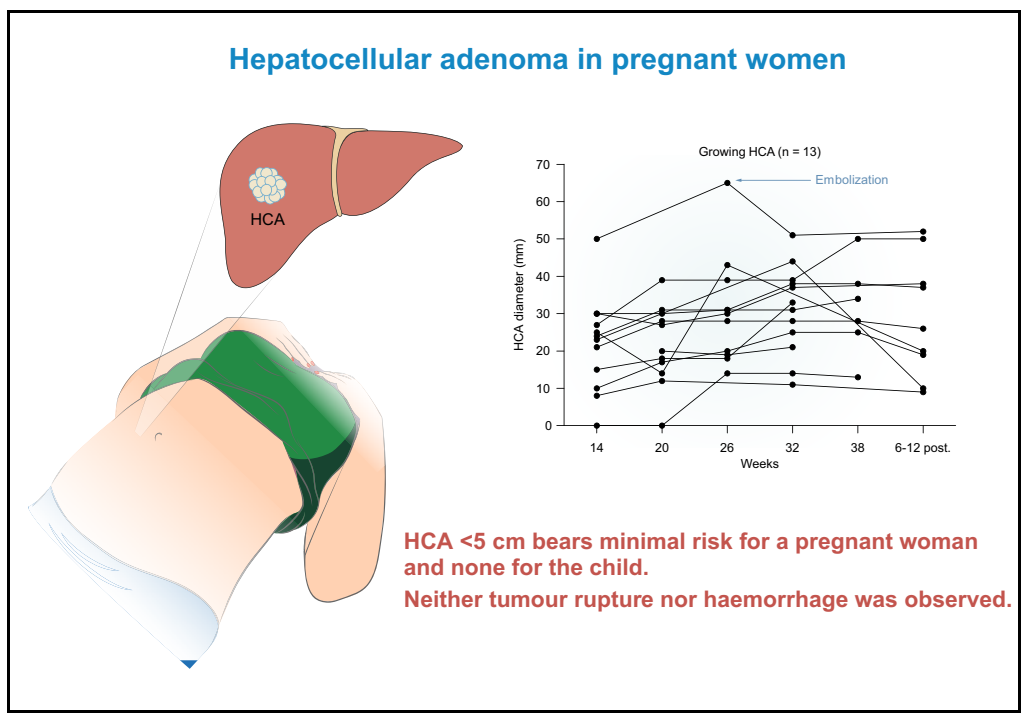

Highlights

- Hepatocellular adenoma $<5 \mathrm{~cm}$ bears minimal risk for a pregnant woman and none for the child.

- In a quarter of cases the hepatocellular adenoma increased in size during pregnancy.

- Neither tumour rupture nor haemorrhage was observed during the study period.

- Close monitoring is recommended in pregnant women with hepatocellular adenoma.

\section{Authors}

Marcia Patricia Gaspersz, Anne Julia Klompenhouwer, Mirelle Elmira Elizabeth Broker, ..., Harry de Koning, Robert Auke de Man, Jan Nicolaas Maria Ijzermans

\section{Correspondence}

j.ijzermans@erasmusmc.nl (J.N.M. Ijzermans)

\section{Lay summary}

The presence of hepatocellular adenoma in pregnant women requires special consideration, as it carries the risk of growth and haemorrhage. In this study we followed 48 patients with hepatocellular adenoma $<5 \mathrm{~cm}$ during 51 pregnancies and found that a hepatocellular adenoma during pregnancy confers minimal risk to the pregnant woman and none to her child. 


\title{
Growth of hepatocellular adenoma during pregnancy: A prospective study
}

\author{
Marcia Patricia Gaspersz ${ }^{1, \dagger}$, Anne Julia Klompenhouwer ${ }^{1, *, \dagger}$, Mirelle Elmira Elizabeth Broker ${ }^{1}$, \\ Maarten Gerardus Josephus Thomeer ${ }^{2}$, Susanna Maria van Aalten ${ }^{1}$, Eric Steegers ${ }^{3}$, \\ Türkan Terkivatan ${ }^{1}$, Harry de Koning ${ }^{4}$, Robert Auke de Man ${ }^{5}$, Jan Nicolaas Maria Ijzermans ${ }^{1}$ \\ ${ }^{1}$ Department of Surgery, Erasmus MC, University Medical Center Rotterdam, The Netherlands; ${ }^{2}$ Department of Radiology, Erasmus MC, \\ University Medical Center Rotterdam, The Netherlands; ${ }^{3}$ Department of Obstetrics and Gynaecology, Erasmus MC, University Medical \\ Center Rotterdam, The Netherlands; ${ }^{4}$ Department of Public Health, Erasmus MC, University Medical Center Rotterdam, The Netherlands; \\ ${ }^{5}$ Department of Gastroenterology and Hepatology, Erasmus MC, University Medical Center Rotterdam, The Netherlands
}

Background \& Aims: The presence of hepatocellular adenoma (HCA) in pregnant women requires special consideration, as it has been reported to carry the risk of growth and clinically significant haemorrhage. In this prospective study we assessed aspects of growth of HCA $<5 \mathrm{~cm}$ during pregnancy.

Methods: This was a multicentre prospective cohort study in pregnant women with suspected HCA $<5 \mathrm{~cm}$ on imaging. Definitive HCA diagnosis was established by MRI with hepatobiliary contrast agents (LCE-MRI), preferably before pregnancy. If at study inclusion a definitive diagnosis was lacking, LCE-MRI was performed after giving birth. Growth of the adenoma (defined as an increase of $>20 \%$ ) was closely monitored with ultrasound examinations throughout pregnancy.

Results: Of the 66 women included, 18 were excluded from analysis because postpartum LCE-MRI did not confirm the diagnosis of HCA and showed the lesion to be focal nodular hyperplasia. The remaining 48 women, with an HCA confirmed by LCE-MRI, were followed during 51 pregnancies. Median age was 30 years (IQR 27-33) and median body mass index $31.9 \mathrm{~kg} / \mathrm{m}^{2}$ (IQR 26.3-36.6). Growth of HCA was seen in 13 of the pregnancies (25.5\%); the median increase was $14 \mathrm{~mm}$ (IQR $8-19$ ). One woman whose HCA grew to $>70 \mathrm{~mm}$ successfully underwent transarterial embolization at week 26 of pregnancy to prevent further growth. The other 50 pregnancies proceeded without complications.

Conclusion: This study suggests that an HCA $<5 \mathrm{~cm}$ confers minimal risk to a pregnant woman and none to her child. HCA increased in size during a quarter of pregnancies, so we recommend close monitoring with ultrasound examinations, enabling intervention if needed. In light of the large proportion of misdiagnosed HCA, LCE-MRI should be performed to prevent unnecessary anxiety in women with a benign liver lesion.

Keywords: Adenoma Liver Cell; Pregnancy; Follow-Up Studies; MRI.

Received 5 July 2019; received in revised form 28 August 2019; accepted 10 September 2019

* Corresponding author. Address: Erasmus MC, University Medical Center Rotterdam, Department of Surgery, Dr. Molewaterplein 40, 3015 GD Rotterdam, The Netherlands. Tel.: +31 107031810 , fax: +31 102250647 .

E-mail address: j.ijzermans@erasmusmc.nl (J.N.M. Ijzermans).

$\dagger$ Shared first authorship.
Lay summary: The presence of hepatocellular adenoma in pregnant women requires special consideration, as it carries the risk of growth and haemorrhage. In this study we followed 48 patients with hepatocellular adenoma $<5 \mathrm{~cm}$ during 51 pregnancies and found that a hepatocellular adenoma during pregnancy confers minimal risk to the pregnant woman and none to her child.

(c) 2019 European Association for the Study of the Liver. Published by Elsevier B.V. All rights reserved.

\section{Introduction}

Hepatocellular adenoma (HCA) occurs particularly among reproductive women and is associated with the use of oestrogen-containing oral contraceptives, androgen intake, obesity, and metabolic disorders. ${ }^{1,2}$ The tumour may regress upon cessation of oestrogen-containing oral contraceptives and weight reduction. ${ }^{3,4}$

Several HCA subtypes can be distinguished radiologically on contrast-enhanced MRI with hepatobiliary contrast agents (LCE$M R I)^{5,6}$ ) or on the basis of immunohistochemical staining or molecular characterization. They include hepatocyte nuclear factor $1 \alpha$ inactivated (H-HCA), inflammatory (I-HCA), $\beta$ catenin-activated ( $\beta$-HCA), $\beta$-catenin-activated inflammatory ( $\beta$-IHCA), and sonic hedgehog (sh-HCA) adenomas. ${ }^{7,8}$ If specific mutations are not found, the HCA is labelled unclassified (UHCA). Resection of an HCA $>5 \mathrm{~cm}$ is usually advocated if it does not regress to $<5 \mathrm{~cm}$ within 12 months, because the risk of complications is thought to be higher in HCA $>5 \mathrm{~cm}^{9-12}$

In pregnant women, HCA requires special attention because of the risk of hormone-induced growth and rupture, which may threaten the life of both mother and child. Cobey and Salem reported that the mortality risk of ruptured HCA $>6.5 \mathrm{~cm}$ during pregnancy was $44 \%$ for the mothers and $38 \%$ for the foetuses [13]. However, almost all cases included in this review dated from the 1970s and 1980s. In 2011, our research group proposed close monitoring of pregnant women with small HCA instead of intervention, and suggested that women with small HCA should not be discouraged to fall pregnant. This suggestion was based on a study in which we monitored 12 women with documented HCA ( 5 with HCA $>5 \mathrm{~cm}$ and 7 with $\mathrm{HCA}<5 \mathrm{~cm}$ ) during a total of 17 pregnancies. ${ }^{14,15}$ All pregnancies in this 
study had an uneventful course without adverse maternal or foetal outcomes.

Data on the behaviour of HCA during pregnancy and the factors that influence safety are still very limited. In this prospective study we assessed the effect of pregnancy on the biological behaviour of HCA, including the incidence of growth, the occurrence of complications and HCA-related interventions during pregnancy. To select a group of women with an a priori low risk of complications, we set the maximum HCA size at $5 \mathrm{~cm}$, as the relevant European guidelines state that HCA $<5 \mathrm{~cm}$ carry low risk of haemorrhage. ${ }^{11}$

\section{Patients and methods \\ Study design and population}

This was a multicentre prospective cohort study performed in the Netherlands, the coordinating centre being a tertiary referral centre for focal liver lesions. The study protocol was reviewed and approved by the institutional review board, has been published before and was registered in the Dutch trial register (NTR3034). ${ }^{16}$

We included pregnant women over 18 years of age who had been diagnosed with single or multiple HCA - the largest not exceeding $5 \mathrm{~cm}$ at the moment of inclusion. Pregnancy had to be confirmed by an obstetrician or midwife with ultrasound (US) examination. Patients were included regardless of parity. The definite HCA diagnosis was based on LCE-MRI or biopsy, preferably performed before pregnancy because contrast agents might be toxic during pregnancy. When the lesion was found incidentally during pregnancy, LCE-MRI was performed postpartum. Exclusion criteria for the per protocol analysis were lack of LCE-MRI or biopsy, no confirmation of the diagnosis HCA on LCE-MRI, and termination of the pregnancy. Inclusion was open from November 2011 until January 2019.

\section{Study procedures}

Eligible women were invited to participate by their treating physicians at the outpatient clinic. Those who were interested were sent a letter explaining the study aims and were contacted by telephone to provide additional information and answer questions. Those who provided written informed consent completed a questionnaire registering: date of birth, length and weight, use of hormonal supplements before pregnancy (including possible fertility treatment), comorbidities, parity and estimated date of delivery.

The participants were scheduled for repetitive US examination at $14(+/-3), 20,26,32,38$ weeks of gestation and 612 weeks postpartum at their preferred hospital. The US examinations were performed by either a radiologist or a hepatologist with experience in hepatic US, who assessed HCA number, location, size, possible growth or regression, as well as US characteristics. Lesion growth was defined as an increase of $>20 \%$ in transversal diameter and lesion regression as a decrease of $>30 \%$ as per the response evaluation in solid tumours (RECIST) criteria. ${ }^{17}$ In case of suspected growth or atypical US characteristics during pregnancy, a conventional MRI (without contrast agents) was performed; the coordinating centre was consulted; and the hepatobiliary multidisciplinary tumour board considered the optimum treatment.

The US reports of participants in the various centres were collected at the coordinating centre. Apart from HCA size at all time points, data on complications during pregnancy, vaginal or caesarean delivery, diagnostic work-up, size of HCA at the time of diagnosis and prior to pregnancy were recorded. In addition, the HCA subtype was documented on the basis of immunohistochemistry or pathomolecular characterization when biopsy material was available, or on LCE-MRI features. ${ }^{5,6,18}$ If haemorrhage had occurred before pregnancy (causing a less reliable subtype determination based on LCE-MRI) or the subtype was not determined otherwise, the subtype remained undetermined ('missing'). The biological behaviour of HCA prior to pregnancy was assessed, that is: regression, growth or stable prior, also according to the RECIST criteria. The percentage of regression was calculated as follows: (diameter HCA at diagnosis - diameter HCA at last follow-up prior to pregnancy)/diameter HCA at diagnosis.

\section{Statistical analysis}

Continuous variables are summarized as mean \pm SD or as median and IQR. Categorical variables are presented as frequency (n) and percentages. Comparative analysis was performed with the Mann-Whitney $U$ test for continuous variables and the $\chi 2$ test for categorical variables. All statistical analysis was performed with SPSS software version 21.0 (IBM, Armonk, New York, USA).

\section{Result \\ Inclusion and baseline characteristics}

A total of 74 pregnant women diagnosed as having an HCA $<5 \mathrm{~cm}$ at the moment of inclusion were scheduled for close monitoring with US during pregnancy. Four refrained from follow-up, however, and 4 had a miscarriage, leaving 66 who completed follow-up. Eighteen women (18/66; 27\%) were excluded from the analysis because LCE-MRI performed after delivery showed the lesion to be focal nodular hyperplasia

Table 1. Baseline characteristics.

\begin{tabular}{|c|c|}
\hline & N (\%) or median (IQR) \\
\hline Age at inclusion $(\mathrm{yr})(\mathrm{n}=51)$ & $30(27-33)$ \\
\hline $\operatorname{BMI}\left(\mathrm{kg} / \mathrm{m}^{2}\right)(\mathrm{n}=51)$ & $31.9(26.3-36.6)$ \\
\hline Diameter HCA at diagnosis $(\mathrm{mm})(\mathrm{n}=48)$ & $33(20-59)$ \\
\hline \multicolumn{2}{|l|}{ Diabetes $(n=48)$} \\
\hline Diabetes mellitus & $3(6.3)$ \\
\hline Gestational diabetes & $3(6.3)$ \\
\hline No & $42(88.8)$ \\
\hline \multicolumn{2}{|l|}{ HCA subtype $(n=48)$} \\
\hline $\mathrm{H}-\mathrm{HCA}$ & $2(4.2)$ \\
\hline I-HCA & $16(33.3)$ \\
\hline U-HCA & $12(25.0)$ \\
\hline Undetermined & $18(37.5)$ \\
\hline \multicolumn{2}{|l|}{ Hormone usage before pregnancy $(n=48)$} \\
\hline Oral contraceptives & $43(89.6)$ \\
\hline None & $4(8.3)$ \\
\hline Other & $1(2.1)$ \\
\hline \multicolumn{2}{|l|}{ Prior pregnancy $(n=51)$} \\
\hline Yes & $12(23.5)$ \\
\hline No & $39(76.5)$ \\
\hline \multicolumn{2}{|l|}{ Fertility treatment $(n=51)$} \\
\hline Yes & $3(5.9)$ \\
\hline No & $48(94.1)$ \\
\hline \multicolumn{2}{|l|}{ Delivery $(n=51)$} \\
\hline Vaginal & $45(88.2)$ \\
\hline Caesarean & $6(11.8)$ \\
\hline
\end{tabular}

Age, BMI and prior pregnancy are given per pregnancy $(n=51)$; the occurrences of diabetes and hormone usage before pregnancy are given per patient $(n=48)$. BMI, body mass index; HCA, hepatocellular adenoma; H-HCA, hepatocyte nuclear factor $1 \alpha$ inactivated HCA; I-HCA, inflammatory HCA; U-HCA, unclassified HCA. 
(FNH) instead of HCA. Ultimately, 51 datasets were analysed as 1 woman participated twice and 1 woman participated 3 times. Baseline characteristics are depicted in Table 1.

In 40 pregnancies $(40 / 51 ; 78 \%)$ the diagnosis HCA was confirmed with LCE-MRI prior to pregnancy. Three patients underwent diagnostic biopsy confirming HCA diagnosis. The median HCA diameter at diagnosis was $33 \mathrm{~mm}$ (IQR 20-59); at the last imaging prior to pregnancy it was $23 \mathrm{~mm}$ (IQR 19-39). In 26 cases, regression had occurred after cessation of oral contraceptives prior to pregnancy; in 14 cases the HCA size had remained stable. The other 11 HCAs were found incidentally during pregnancy.

\section{Follow-up during pregnancy}

All 5 scheduled US examinations were performed in $18 \%$ of the 51 pregnancies; 4 examinations were performed in 54\%; 3 examinations were performed in $22 \%$; and 2 examinations were performed in $6 \%$. Reasons for missing examinations were inclusion after 14 weeks of pregnancy, delivery prior to 38 weeks and

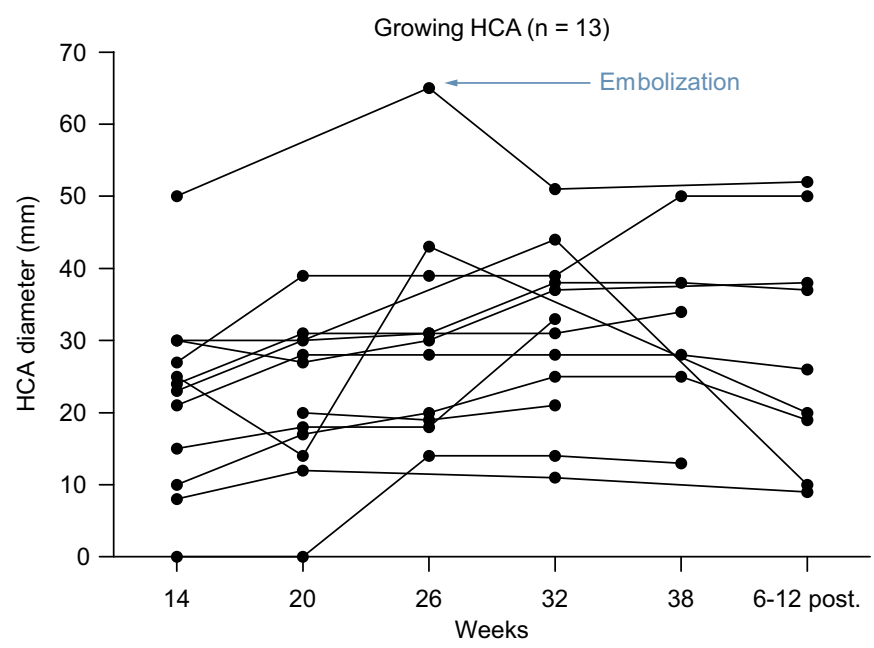

Fig. 1. Growing HCA during pregnancy. This figure shows the 13 growing HCA during pregnancy at each time point: $14(+/-3), 20,26,32,38$ weeks of gestation and 6-12 weeks postpartum). HCA, hepatocellular adenoma. patient non-compliance. Postpartum US was performed in $70 \%$ and showed either stable or regressing lesions.

In 27 of the 51 pregnancies (53\%), the lesions remained stable; in 11 (22\%) they had regressed, and in 13 (25\%) they had grown. In all 13 cases, growth had occurred between 14 and 32 weeks of gestation; the median growth was $14 \mathrm{~mm}$ (IQR 8-19) (Fig. 1). During pregnancy, HCA imaging indicating bleeding was not found and lesion characteristics did not differ between the subgroup of women with stable or regressing HCA and the subgroup of women with growing lesions ( $p=0.249$, Table 2). Additionally, there were no statistically significant differences between these subgroups for age at inclusion, body mass index (BMI) at inclusion, HCA diameter at diagnosis, HCA subtype and the percentage of HCA regression before pregnancy (Table 2). One woman underwent a transarterial embolization of the HCA during pregnancy because it had grown from $49 \mathrm{~mm}$ at week 14 to a maximum of $76 \mathrm{~mm}$ at week 20 (Fig. 2). She had been diagnosed with HCA 2 years prior to pregnancy; at baseline it measured $93 \mathrm{~mm}$ and it regressed to $49 \mathrm{~mm}$ within 1 year after cessation of oral contraceptives (Fig. 3). After the embolization, the lesion regressed to $65 \mathrm{~mm}$ at week 26 and $51 \mathrm{~mm}$ at week 32. Labour was induced at 38 weeks, proceeded without complications, and resulted in the birth of a healthy child. The other 50 pregnancies proceeded without complications. Six patients had a caesarean delivery, all because of reasons unrelated to HCA. The remaing deliveries were vaginal with an uneventful course.

Three patients underwent fertility treatment in order to become pregnant, of whom 2 underwent in vitro fertilisation and 1 ovulation induction. Although no US examinations were performed during the fertility therapy and therefore possible growth of HCA was not monitored, clinically, the fertility treatment was described as an uneventful course. During pregnancy, the HCA showed growth in 1 patient and remained stable in the other 2 .

Two women were enrolled in the study more than once, respectively 2 and 3 times. Both underwent LCE-MRI after each pregnancy. The former had a lesion of $20 \mathrm{~mm}$ that remained stable during the first pregnancy, and then regressed in the 2 years until the second pregnancy to a point where it was not visible anymore. During the second pregnancy it grew to a maximum of $13 \mathrm{~mm}$. The latter had a lesion that remained

Table 2. Comparison of growing vs. stable/regressing lesions during pregnancy.

\begin{tabular}{|c|c|c|c|}
\hline & Stable or regression $(n=38)$ & Growth $(n=13)$ & $p$ value \\
\hline Age at inclusion (yr) & $29(27-32.3)$ & $32(29-34)$ & 0.155 \\
\hline BMI $\left(\mathrm{kg} / \mathrm{m}^{2}\right)$ & $32.3(26.1-37.6)$ & $29.0(27.1-35.9)$ & 0.603 \\
\hline Diameter HCA at diagnosis (mm) & $35.0(20.0-64.0)$ & $30.0(20.0-60.0)$ & 0.952 \\
\hline HCA subtype & & & 0.540 \\
\hline $\mathrm{H}-\mathrm{HCA}$ & $2(5.3)$ & $0(0)$ & \\
\hline $\mathrm{I}-\mathrm{HCA}$ & $12(31.6)$ & $4(30.8)$ & \\
\hline U-HCA & $12(31.6)$ & $2(15.4)$ & \\
\hline Undetermined & $12(31.6)$ & $7(53.8)$ & \\
\hline Percentage regression of HCA before pregnancy & $35.9(13.9-59.5)$ & $47.3(0-73.0)$ & 0.858 \\
\hline Biological behaviour of HCA prior to pregnancy & & & 0.249 \\
\hline Regression & $23(60.5)$ & $8(61.5)$ & \\
\hline Stable & $5(13.2)$ & $4(30.8)$ & \\
\hline Unknown & $9(23.7)$ & $2(15.4)$ & \\
\hline
\end{tabular}

Comparative analysis was performed with Mann-Whitney $U$ test for continuous variables and $\chi 2$ test for categorical variables. The percentage of regression of HCA before pregnancy was calculated as followed: (diameter HCA at diagnosis - diameter HCA at last follow-up prior to pregnancy)/diameter HCA at diagnosis. The biological behaviour of HCA prior to pregnancy was assessed and it was documented whether the lesion regressed or was stable by the RECIST criteria. BMI, body mass index; HCA, hepatocellular adenoma; H-HCA, hepatocyte nuclear factor $1 \alpha$ inactivated HCA; I-HCA, inflammatory HCA; U-HCA, unclassified HCA. 

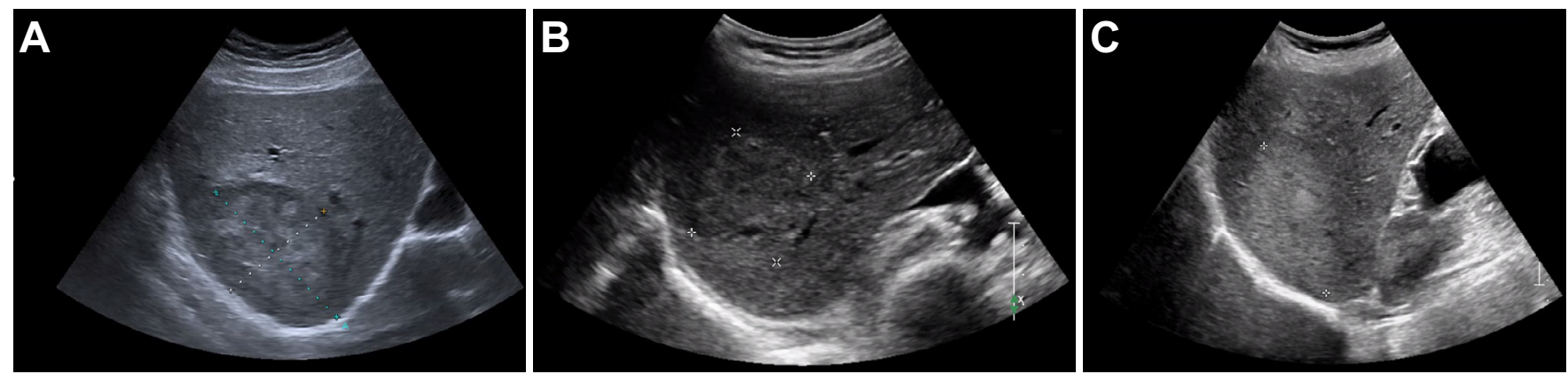

Fig. 2. Ultrasound of growing HCA. Patient who was diagnosed with a single HCA of $93 \mathrm{~mm}$ (A: ultrasound at diagnosis). After cessation of oral contraceptives the lesion regressed to $49 \mathrm{~mm}$ (B: last ultrasound prior to pregnancy). During pregnancy the lesion showed growth again to a maximum of 76 mm (C: ultrasound showing growth during pregnancy). A transarterial embolization was performed. HCA, hepatocellular adenoma.
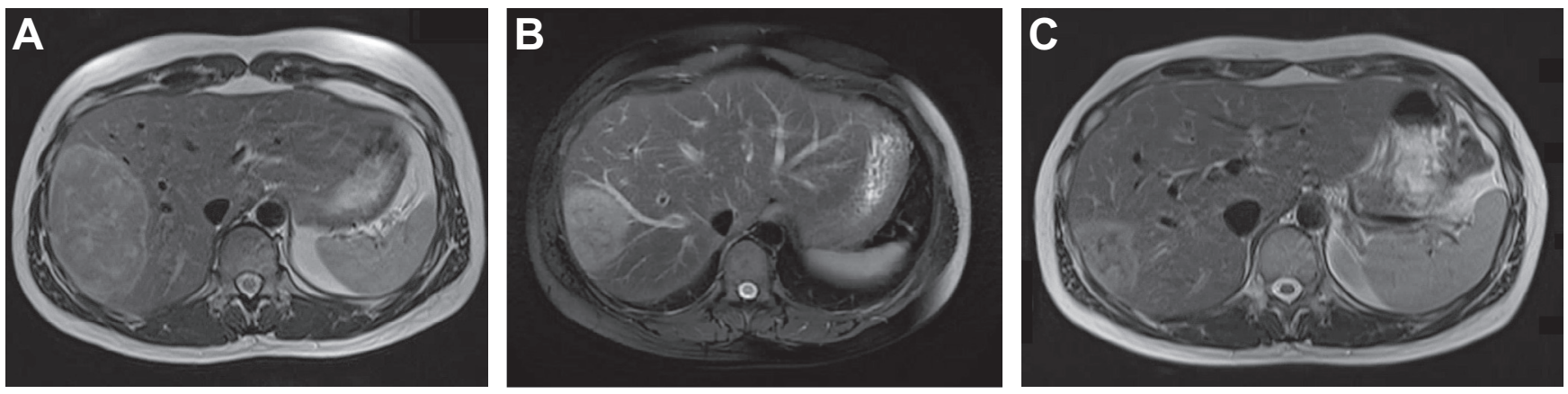

Fig. 3. MRI of growing HCA. T2 weighted MRI images of a patient with a larger single HCA. (A) Diagnostic contrast-enhanced MRI showing a large HCA $(93 \mathrm{~mm}$ ) in the right hemiliver. (B) MRI performed during pregnancy due to suspected growth, showing a $65 \mathrm{~mm}$ lesion. As the lesion grew significantly early on in the pregnancy, transarterial embolization was performed. (C) MRI postpartum, showing a $52 \mathrm{~mm}$ lesion with central necrosis after transarterial embolization. HCA, hepatocellular adenoma.

stable at $43 \mathrm{~mm}$ during the first and stable at $25 \mathrm{~mm}$ during the second pregnancy, while it regressed during the third pregnancy.

Five women had been diagnosed with haemorrhagic HCA prior to pregnancy; all were hemodynamically stable and intervention was judged unnecessary. The HCA grew during pregnancy in 2 women and remained stable in the other 3 . Neither of the lesions showed signs of haemorrhage during pregnancy.

None of the 18 women who were diagnosed with FNH postpartum had undergone LCE-MRI prior to pregnancy in the hospital where they were diagnosed. Suspicion of HCA had been raised during conventional MRI (without hepatobiliary contrast agent) in 12, US in 4, CT in 1 and contrast-enhanced US in 1.

\section{Discussion}

In this study of 51 pregnancies in 48 patients with $\mathrm{HCA}<5 \mathrm{~cm}$, we investigated the incidence of growth, occurrence of complications and HCA-related interventions during pregnancy. HCA growth was defined as an increase of more than $20 \%$, as per the RECIST criteria, and this was seen in one-quarter of cases. The median growth was $14 \mathrm{~mm}$. Neither tumour rupture nor haemorrhage was observed during the study period, and factors predictive of growth could not be identified.

To the best of our knowledge, this is the first prospective study investigating the biological behaviour of small HCA during pregnancy. Until recently, the general consensus had been that women with HCA should be discouraged to become pregnant without prior treatment - in view of the risk of clinically relevant or even life-threatening haemorrhage. Furthermore, in a paper studying mainly cases from the 1970s and 1980s it was reported that during pregnancy the mortality risk of ruptured HCA may be as high as $44 \%$ for the mother and $38 \%$ for the foetus. ${ }^{13}$ It should be noted that this was at a time in which the entity of HCA was less well-known and embolization for acute haemorrhage was not yet available in all hospitals.

Only in 1 case, in which rapid growth of the lesion during pregnancy was seen, was an intervention performed to reduce the risk of haemorrhage. This patient had previously been diagnosed with a large HCA that had regressed to $<5 \mathrm{~cm}$ within 1 year after cessation of oral contraceptives. The intervention consisted of transarterial embolization; labour was uncomplicated and resulted in the birth of a healthy child. As other complications did not occur in this cohort, we suggest that women with HCA $<5 \mathrm{~cm}$ who wish to conceive should not be discouraged or advised to refrain from this. Still, in view of the fact that growth occurred in one-quarter of cases, shared decision making with the patient and close monitoring during pregnancy is indicated. We surmised that HCAs that regress after cessation of oral contraceptives are more sensitive to hormones - and therefore are more prone to grow during pregnancy. However, we could not confirm this with the present data, probably because of the lack of statistical power resulting from the small sample size. Likewise, we could not detect differences between HCA subtypes, although previous studies have shown different probabilities of HCA regression and risk of complication for various HCA subtypes. ${ }^{8,19}$ Surprisingly, almost one-quarter of HCAs 
in this study regressed during pregnancy. The exact mechanism and reason for this regression remains unclear.

In this study, only 3 patients underwent fertility treatment in order to become pregnant. No US examinations were performed during fertility treatment as these patients were only included after conception, but fertility treatment was described as uneventful. Indeed, it would be interesting to monitor the size of HCA during fertility treatment and its hormonal stimulation and to relate the outcome with changes during pregnancy. Future studies should aim to investigate the effect of fertility treatment on HCA.

Two women in this study were enrolled more than once: 1 woman during 2 pregnancies and 1 woman during 3 pregnancies. HCA behaviour was not the same in the consecutive pregnancies. An additional 9 women already had had an uneventful pregnancy before being diagnosed with HCA, and all had uneventful pregnancies again during this study. By way of comparison, in a previous study almost half of the women with HCA had already carried at least 1 pregnancy to term before diagnosis. $^{20}$

The median BMI at inclusion of patients enrolled in this study is fairly high. An association between HCA and obesity has been well established in the literature., ${ }^{4,21-23}$ Additionally, the median BMI in this study is comparable to the median of previous study populations. ${ }^{12,19}$ Another notable result in the baseline characteristics is the high fraction of patients with UHCA, especially compared to previously published studies. ${ }^{7}$ Previous studies regarding the distribution of HCA subtypes have particularly been performed in cohorts of patients with larger adenomas. An analysis regarding HCA subtype related to the size has yet to be performed, but subtype distribution might differ between large and small HCAs. Additionally, biopsy is less often performed when the lesion is small.

In the present study, a large proportion of suspected HCAs actually proved to be FNH. Such a misdiagnosis could have a major impact on the life of the young woman, as FNH is not associated with hormone-induced growth and the lesion need not be closely monitored during pregnancy. Therefore, we highly recommend performing LCE-MRI if HCA is suspected and the woman wishes to conceive.

Regrettably, we had to deviate from the original study protocol of the PALM study as published in 2012. ${ }^{16}$ This included 2 control groups for the purpose of assessing quality of life aspects: one of healthy pregnant women without HCA and one of pregnant women with diabetes mellitus. Because we could not find women without HCA willing to undergo regular liver US during pregnancy, these 2 control groups were discarded. Venipuncture, as described in the protocol, was discarded as well, because the majority of participants were unwilling to undergo venipuncture.

This study is subject to some limitations. First, only $18 \%$ of participants underwent the US examination at every planned follow-up moment. The incomplete data do not, however, affect the primary study outcome. Second, the study cohort was too small to identify with a high degree of certainty risk factors for rupture, bleeding or growth. Nevertheless, considering the rareness of this liver tumour, the sample size was relatively large. Future studies should aim to identify risk factors for pregnancy-related haemorrhage or growth of HCA. Lesion characteristics such as HCA subtype or HCA response after cessation of oral contraceptives might affect the risk of rupture or bleeding. ${ }^{8}$
International collaborations are needed to create a study cohort large enough to identify subgroups at risk of pregnancy-related complications. Taking biopsies could help to identify HCA subtypes and define women at risk who may benefit from follow-up and those who do not require followup due to the very low risk of complications. To gain more insight into the accuracy of diagnostics, the biological behaviour of HCA during pregnancies, and the risk of growth and haemorrhage of HCA during pregnancy, we initiated the EuroPALM registry, which will enable the field to create a large international cohort.

In conclusion, this study indicates that in well-diagnosed patients, an HCA smaller than $5 \mathrm{~cm}$ during pregnancy seems to bear minimal risk for the mother and no risk for the foetus. As it cannot be excluded that an HCA will grow during pregnancy, we recommend close monitoring with ultrasound examinations.

\section{Abbreviations}

$\beta$-HCA, $\beta$-catenin-activated HCA; $\beta$-IHCA, $\beta$-catenin-activated inflammatory HCA; BMI, body mass index; FNH, focal nodular hyperplasia; HCA, hepatocellular adenoma; H-HCA, hepatocyte nuclear factor $1 \alpha$ inactivated HCA; I-HCA, inflammatory HCA; sh-HCA, sonic hedgehog HCA; LCE-MRI, MRI with hepatobiliary contrast agents; U-HCA, unclassified HCA; US, ultrasound.

\section{Financial support}

For this study we received financial support from 2 foundations: the Nuts Ohra Fonds, Amsterdam, the Netherlands and Stichting Coolsingel, Rotterdam, the Netherlands.

\section{Conflict of interest}

The authors declare no conflicts of interest that pertain to this work.

Please refer to the accompanying ICMJE disclosure forms for further details.

\section{Authors' contributions}

Study design: SMvA, ES, TT, HdK, RAdM, JNMI. Data collection: MPG, AJK, MEEB, MGJT, SMvA. Data analysis and interpretation: MRG, AJK, RAdM, JNMI. Drafting of the manuscript: MPG, AJK, RAdM, JNMI. Critical revision of the manuscript: MMEB, MGJT, SMvA, ES, TT, HdK, RAdM, JNMI.

\section{Acknowledgements}

Erasmus MC Rotterdam, The Netherlands: D. Sprengers, P. Taimr, R.J. de Knegt.

Gelre Ziekenhuizen, The Netherlands: K. Hack, E. Kouw, G. Semplonius. Medisch spectrum Twente, The Netherlands: N. Venneman, M. Guichelaar, J.M. Klaase, A. Hulsegge. Deventer Ziekenhuis, The Netherlands: J. Nieuwenhuis-Scholthof, M.S.L. Liem, M.E. Bartelink. Radboud UMC, The Netherlands: E. Tjwa, H. de Wilt, M. Koppe.

Flevoziekenhuis, The Netherlands: H. Telleman. Zuyderland Ziekenhuis, The Netherlands: M. Romberg-Camps, K. Soufidi. Maxima Medisch Centrum, The Netherlands: S. Goossens. Alrijne Ziekenhuis, The Netherlands: S. Abraham. 
OLVG, The Netherlands: M. van Ierland-van Leeuwen. Diakonessenhuis, The Netherlands: A. Voorburg. Franciscus Gasthuis en Vlietland, The Netherlands: I. Leeuwenburgh. Isala Ziekenhuis, The Netherlands: G. Patijn, J. Haanstra. Rijnstate Ziekenhuis, The Netherlands: M. Spanier.

\section{Supplementary data}

Supplementary data to this article can be found online at https://doi.org/10.1016/j.jhep.2019.09.011.

\section{References}

Author names in bold designate shared co-first authorship

[1] Reddy KR, Schiff ER. Approach to a liver mass. Semin Liver Dis 1993:13:423-435.

[2] Rooks JB, Ory HW, Ishak KG, Strauss LT, Greenspan JR, Hill AP, et al. Epidemiology of hepatocellular adenoma. The role of oral contraceptive use. JAMA 1979;242:644-648.

[3] Edmondson HA, Reynolds TB, Henderson B, Benton B. Regression of liver cell adenomas associated with oral contraceptives. Ann Intern Med 1977;86:180-182.

[4] Dokmak S, Belghiti J. Will weight loss become a future treatment of hepatocellular adenoma in obese patients? Liver Int 2015;35:2228-2232.

[5] Ronot M, Bahrami S, Calderaro J, Valla DC, Bedossa P, Belghiti J, et al. Hepatocellular adenomas: accuracy of magnetic resonance imaging and liver biopsy in subtype classification. Hepatology 2011;53:1182-1191.

[6] van Aalten SM, Thomeer MG, Terkivatan T, Dwarkasing RS, Verheij J, de Man RA, et al. Hepatocellular adenomas: correlation of MR imaging findings with pathologic subtype classification. Radiology 2011;261:172-181.

[7] Nault JC, Bioulac-Sage P, Zucman-Rossi J. Hepatocellular benign tumorsfrom molecular classification to personalized clinical care. Gastroenterology 2013;144:888-902.

[8] Nault JC, Couchy G, Balabaud C, Morcrette G, Caruso S, Blanc JF, et al. Molecular classification of hepatocellular adenoma associates with risk factors, bleeding, and malignant transformation. Gastroenterology 2017;152(880-894) e886.

[9] van Aalten SM, de Man RA, IJzermans JN, Terkivatan T. Systematic review of haemorrhage and rupture of hepatocellular adenomas. Br J Surg 2012;99:911-916.
[10] Stoot JH, Coelen RJ, De Jong MC, Dejong CH. Malignant transformation of hepatocellular adenomas into hepatocellular carcinomas: a systematic review including more than 1600 adenoma cases. HPB (Oxford) 2010;12:509-522.

[11] European Association for the Study of the L. EASL Clinical Practice Guidelines on the management of benign liver tumours. J Hepatol 2016;65:386-398.

[12] Klompenhouwer AJ, Broker MEE, Thomeer MGJ, Gaspersz MP, de Man RA, Ijzermans JNM. Retrospective study on timing of resection of hepatocellular adenoma. Br J Surg 2017;104:1695-1703.

[13] Cobey FC, Salem RR. A review of liver masses in pregnancy and a proposed algorithm for their diagnosis and management. Am J Surg 2004;187:181-191.

[14] Noels JE, van Aalten SM, van der Windt DJ, Kok NF, de Man RA, Terkivatan T, et al. Management of hepatocellular adenoma during pregnancy. J Hepatol 2011;54:553-558.

[15] Broker ME, Ijzermans JN, van Aalten SM, de Man RA, Terkivatan T. The management of pregnancy in women with hepatocellular adenoma: a plea for an individualized approach. Int J Hepatol 2012;2012 725735.

[16] van Aalten SM, Broker ME, Busschbach JJ, de Koning HJ, de Man RA, Steegers EA, et al. Pregnancy and liver adenoma management: PALMstudy. BMC Gastroenterol 2012;12:82.

[17] Eisenhauer EA, Therasse P, Bogaerts J, Schwartz LH, Sargent D, Ford R, et al. New response evaluation criteria in solid tumours: revised RECIST guideline (version 1.1). Eur J Cancer 2009;45:228-247.

[18] Laumonier H, Bioulac-Sage P, Laurent C, Zucman-Rossi J, Balabaud C, Trillaud H. Hepatocellular adenomas: magnetic resonance imaging features as a function of molecular pathological classification. Hepatology 2008;48:808-818.

[19] Klompenhouwer AJ, Alblas M, Van Rosmalen BV, Haring MPD, Venema E, Doukas M, et al. Development and validation of a model to predict regression of large size hepatocellular adenoma. Am J Gastroenterol 2019.

[20] van der Windt DJ, Kok NF, Hussain SM, Zondervan PE, Alwayn IP, de Man $\mathrm{RA}$, et al. Case-orientated approach to the management of hepatocellular adenoma. Br J Surg 2006;93:1495-1502.

[21] Bioulac-Sage P, Taouji S, Possenti L, Balabaud C. Hepatocellular adenoma subtypes: the impact of overweight and obesity. Liver Int 2012:32:1217-1221.

[22] Bunchorntavakul C, Bahirwani R, Drazek D, Soulen MC, Siegelman ES, Furth EE, et al. Clinical features and natural history of hepatocellular adenomas: the impact of obesity. Aliment Pharmacol Ther 2011;34:664-674.

[23] Broker MEE, Gaspersz MP, Klompenhouwer AJ, Hansen BE, Terkivatan T, Taimr P, et al. Inflammatory and multiple hepatocellular adenoma are associated with a higher BMI. Eur J Gastroenterol Hepatol 2017;29:1183-1188. 